\title{
International Collaboration through the Volunteer Lecturer Program
}

\author{
Padmanabhan Seshaiyer
}

Communicated by Harriet Pollatsek

ABSTRACT. I describe an international program in which faculty teach intensive mathematics courses at the advanced undergraduate or master's level in a developing country. I also describe the impact on me and on the scientific and professional growth of other faculty and students over the last several years.

In the spring of 2011, I was selected for the Volunteer Lecturer Program (VLP) administered through the Commission for Developing Countries (CDC) of the International Mathematical Union to serve at the Nelson Mandela African Institute of Science and Technology in Arusha, Tanzania. I was invited to provide a 4-week intensive course on numerical analysis and mathematical modeling for graduate students.

The CDC identifies mathematicians interested in contributing to the education of young mathematicians in the developing world. It maintains a VLP database [1] listing each mathematician's curriculum vitae, areas of mathematics, language abilities, typical dates of availability, and previous experience. The CDC also identifies appropriate universities and mathematics degree programs in the developing world. VLP lecturers offer 3- to 4 -week mathematics courses in topics at the advanced undergraduate and graduate levels. Two main objectives of the VLP are to build capacity in mathematics and mathematics education in developing countries and to increase interaction between the mathematical community in the developed world and the vast, mostly untapped reser-

Padmanabhan Seshaiyer (Padhu) is professor of mathematical sciences at George Mason University. He serves as the associate dean for the College of Science and the director for the Center for Outreach in Mathematics Professional Learning and Educational Technology (COMPLETE).

His email address is pseshaiy@gmu.edu and his website is http://math.gmu.edu/ pseshaiy/outreach.htm1.

For permission to reprint this article, please contact: reprint-permission@ams . org.

DOI: http://dx.doi.org/10.1090/noti1711

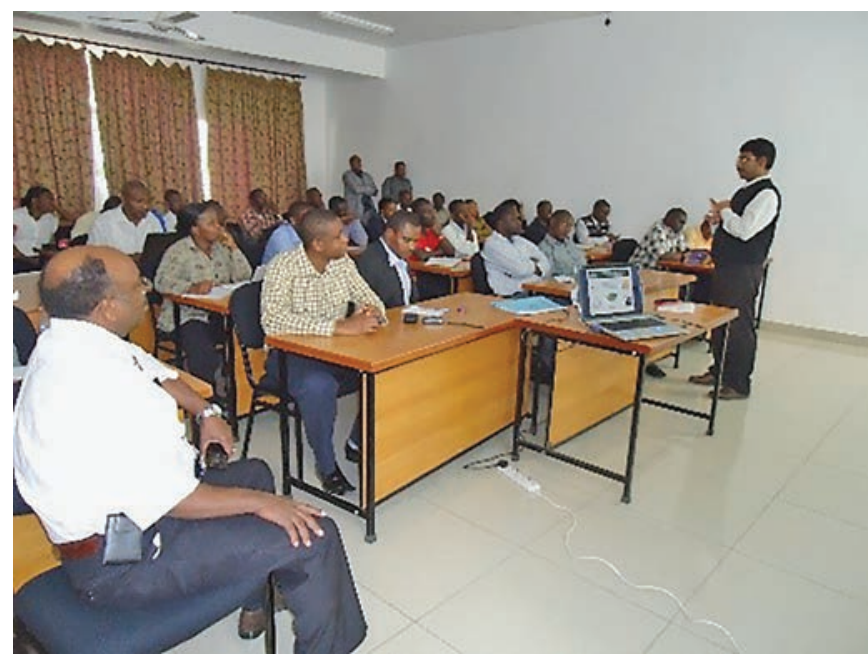

Figure 1. When my students failed to arrive, I began my work at the Mandela Institute teaching the teachers.

voir of mathematical talent in the developing world. The program receives financial support from the American Mathematical Society, the National Academy of Sciences, and the Abel Board.

The involvement of US mathematicians in the VLP began with an appeal from the AMS to US mathematics department chairs. From 2009 to 2014, US mathematicians delivered at least 21 intensive short courses for graduate programs at universities in seven countries in Asia, Central America, and Africa. More details about the VLP and how to apply can be found at their website [1]. 


\section{My Personal VLP Experience}

The program gave me a great opportunity to learn from faculty and students at Mandela Institute, and I had to be ready for unexpected challenges. Challenges in the developing world can involve communication, governmental protocols, availability of electricity, students' lack of background knowledge, and many more. I came to appreciate Albert Einstein's statement, "The measure of intelligence is the ability to change." Let me explain.

When I landed in Arusha on the Wednesday prior to my beginning week of teaching, the Deputy Vice Chancellor for Research received me. I enjoyed a warm welcome and was happy to learn that I was their first visiting lecturer from the US. The first cohort of students was supposed to arrive that weekend to start classes on the following Monday. My excitement only lasted for a day, until I found out that the government had to postpone the start date of the university by two months, so the students would arrive after my departure, and the situation was out of the university's control. So there I was in Arusha, Tanzania, not knowing what to do for the next four weeks. But I did not give up hope. I learned that the entire NM-AIST faculty, who were both master's and doctoral degree holders from various disciplines, were already working on campus. So the then Vice-Chancellor (equivalent to the President of a US institution) Prof. Burton Mwamila and I decided to create a Train the Trainers program, as he felt that all of his faculty needed both mathematical training and professional development. Besides providing them a good exposure in numerical analysis and mathematical modeling, I offered several faculty development workshops on leadership, curriculum development, project management, academic affairs, and best practices for institutional transformation. So my first university students were many of the faculty, who were very happy to be a part of my VLP (see Figure 1).

\section{My Second Visit}

The relationship that I developed with the institute as well as the collaboration with faculty and some other activities were very valuable, and I was encouraged to visit the Mandela Institute again as their 2012 VLP lecturer. This time, they already had students. I developed an introductory mathematical modeling and scientific computing course employing MATLAB and OCTAVE, which all future

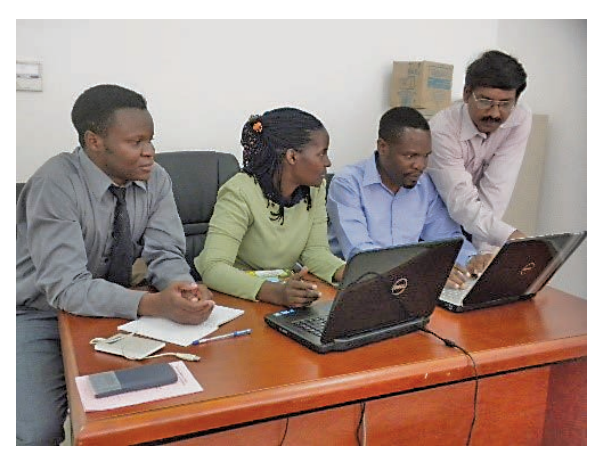

Figure 2: I taught first-year graduate students to program numerical methods for their group project.
I gave lectures on creating effective research proposals and helped many students to come up with focused research topics for their masters or PhD. Figure 2 shows a session with three first-year students in my office to learn to program numerical methods for their group project. I was happy to engage the students in interdisciplinary collaborative research opportunities that helped to promote their awareness of the applications of mathematics to solve problems of national importance in areas such as agriculture, food-security, bio-diversity, mobile-medicine, e-learning, disease modeling and prevention, waste management, water resources, and fish harvesting. The university at the same time appointed me to an adjunct professorship, so I was able to direct students and work with the institution on joint proposals.

The VLP experience also gave me the opportunity to help Tanzania win their first ever Partnership for Enhanced Engagement in Research (PEER) Science grant. PEER Science is a competitive grants program that invites scientists in developing countries to apply for funds to support research and capacity-building activities on topics of importance to the United States Agency for International Development. The grant is coordinated in partnership with many other agencies in the US, including NASA, NIH, NOAA, NSF, USDA, USGS, and the Smithsonian Institution. My work with Tanzania researchers was a perfect fit for this program and led to the country applying for and winning the PEER Science award in phase 2 of the program, which was administered by the National Academies. The program included me as the US collaborator on creating a new PEER Science research program on Computational Mathematics, Modeling and Analysis of Biological, Bio-inspired and Engineering Systems. ${ }^{1}$ The project has helped to increase STEM capacity in Tanzania through research in food security, environment, education, water, and global health. Through these projects, the participants were able to work directly with governmental agencies in Tanzania to collect data that helped to validate their computational model and to create better infrastructure. The project

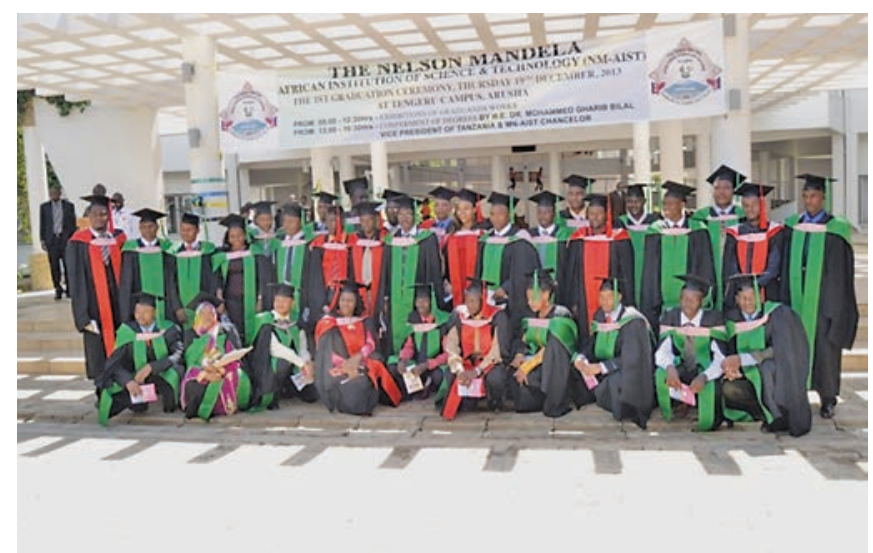

Figure 3: Graduate students worked with government agencies in Tanzania on their model for better infrastructure (2013).

1 sites nationalacademies.org/PGA/PEER/PEERscience/ PGA_084056. 
offered support to more than 20 graduate students who went on to pursue their masters or PhD degrees (see Figure 3). This has not only been a huge achievement for NM-AIST and Tanzania, but also has helped the Tanzanian government make informed health, educational, and public policy decisions in the country.

\section{Tracking poachers}

The collaborations and partnerships that the VLP helped to establish have led to several research and outreach initiatives. In 2015, I led a group of researchers including faculty, graduate and undergraduate students, and high school teachers and students from the US and Tanzania investigating the problem of poaching of elephant tusks and rhino horns. The team identified observation by drones as a solution. The research required them to understand mathematically modeling drone dynamics. Moreover, to find poachers, we proposed using a Bayesian framework as the search pattern continues through the

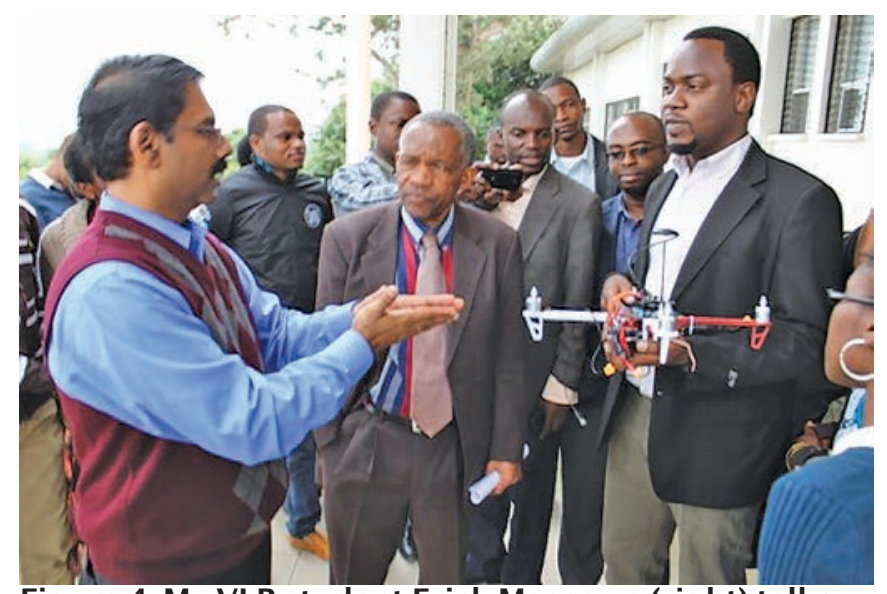

Figure 4. My VLP student Erick Massawe (right) talks with me about tracking poaching as Vice-Chancellor Mwamila (center) looks on.

evolution of a belief function. This function was generated through a sequence of observations that allowed for the computation of individual belief probabilities to iteratively identify target locations. This project went on to be one of the 60 projects selected from hundreds of applications to be showcased at the Council on Undergraduate Research Posters on the Hill event in Washington, D.C. in 2016 [2, 3]. My VLP student Erick Massawe (Figure 4) is completing his $\mathrm{PhD}$ work on developing intelligent tracking systems to stop poaching. ${ }^{2}$ Currently, I am directing another PhD student at the institute, who was a part of a VLP program to develop a mathematical model addressing health risks associated with alcoholism in the presence of religious beliefs in Tanzanian communities. Several of these projects involve mathematical modeling and solutions to systems of coupled non-linear differential equations taught in the VLP course $[4,5]$.

2 See the Mathematical Moment on "Thwarting Poachers" www. ams.org/publicoutreach/mathmoments/mm122-poaching-podcast.

\section{How the Program Changed $\mathrm{Me}$}

This VLP opportunity helped me to create successful pathways for teaching, research, and service for both US mathematicians and their counterparts in the developing world. Many VLP lecturers like me mentor their students and supervise their MS theses during their visits. Some of them continue these tasks through weekly or monthly virtual meetings after they return to the US. Helen Tyler (Manhattan College), one of the volunteers in Cambodia, said:
I have learned so much from my interactions with the Cambodian mathematical community. The students have been the hardest working and hungriest that I have ever taught. And it is possible that I have learned even more from them. At home, my students have become al- most too familiar; I am rarely asked a question that I have not been previously asked. But here my students come to the material with a differ- ent set of skills, some even stronger than my students at home. And so I feel more present during my lectures here than I often do in the United States. I am more sharply focused on how the students react to the material and to how I present it. I am certain that the experi- ence has made me a better teacher, both here and at home.

The VLP program has definitely transformed my academic career as it has given me the opportunity to engage in an educational philosophy that promotes development of life-long learning skills including communication, collaboration, critical thinking, and creativity through mathematics. It has continued to help me to practice and preach about finding the mathematics to solve a given real-world problem rather than looking for a real-world problem to apply the mathematics. I currently have students working on poaching in Africa; Zika prevention strategies in Ecuador; the spread of gangs in Puerto Rico; the social dynamics of adults and children involved in trafficking between Latin America, Mexico, and the US; modeling spread of waterborne diseases in networks in India; and identifying landmines in Colombia. 


\section{Advertise in the Notices}

of the American Mathematical Society

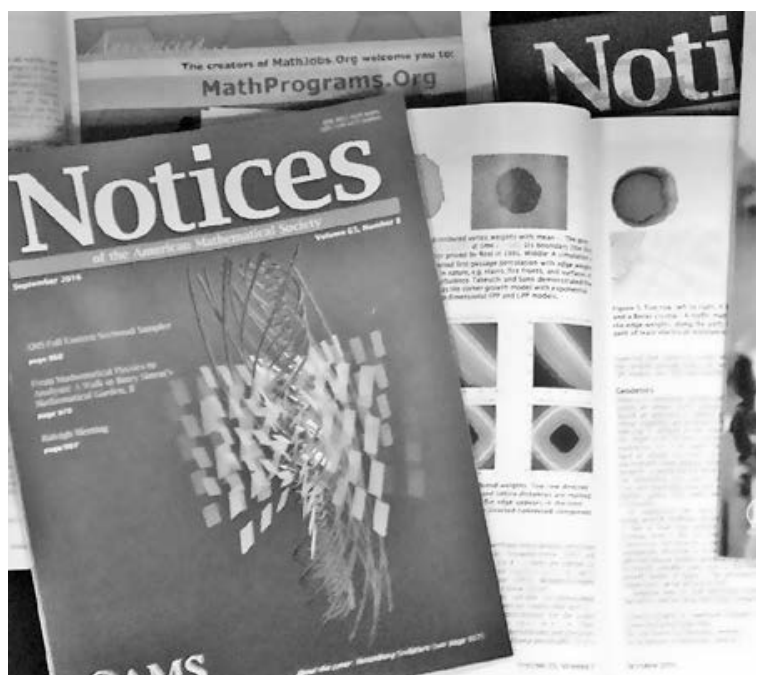

Connect with an audience of approximately 30,000 subscribers through Classified and Display Advertising in the Notices of the American Mathematical Society, available in print and online.

As the world's most widely read magazine aimed at professional mathematicians, the Notices is an excellent medium for announcing publications, products, and services, and for recruiting mathematical scientists in academic, industry, and government.

Learn more about advertising in the Notices at

www.ams.org/noticesadvertising.

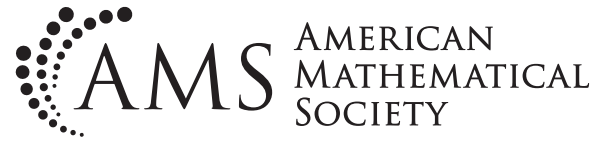

\section{COMMUNICATION}

\section{References}

[1] UNIFORM RESOURCE LOCATOR: https: //www . mathunion . org /cdc/lecturing/volunteer-1ecturer-program.

[2] BAEZ, A., MCLANE, K., \& PRADYUTA, P. (2016, April). Simulation and visualization of quadcopter flight dynamics with applications to search and rescue operations, Council on Undergraduate Research: 20th Annual Posters on the Hill. Washington, DC.

[3] Pearl J.S. AND Seshaiyer, P. (2017 April). Applying design thinking to mathematics research: developing an interdisciplinary method to combat poaching, SIAM News. https://sinews.siam.org/Details-Page/applying-deSign-thinking-to-mathematics-research-2.

[4] P. SESHAIYER (2017) Leading undergraduate research projects in mathematical modeling, PRIMUS, 27(4-5), 476-493, DOI: 10.1080/10511970.2016.1240732.

[5] P. SESHAIYER (2012), Transforming practice through undergraduate research, CUR FOCUS, Council on Undergraduate Research, 33(1).

\section{Photo Credits}

All photos courtesy of the author.

ACKNOWLEDGEMENT: The author acknowledges senior program officers at the National Academies Ana Ferreras and Dalal Najib, who coordinate programs in which he serves.

\section{ABOUT THE AUTHOR}

Padhu Seshaiyer's research interests are in computational mathematics, scientific computing, computational biomechanics, and STEM education. He has initiated and directed a variety of educational programs including graduate and undergraduate research, K-12 outreach, and teacher professional development. He serves as the chair of the Diversity Advisory Committee for the Society for Industrial and Applied Mathematics.

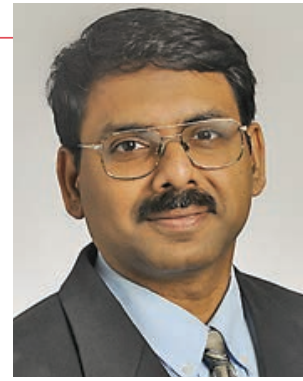

Padhu Seshaiyer 